\title{
Grand Challenges in Photonics: Route to Light
}

\author{
Marco Peccianti * \\ Emergent Photonics (EPic) Laboratory, Department of Physics and Astronomy, University of Sussex, Brighton, United Kingdom
}

Keywords: photonics in economy, photonics in society, interdisciplinary photonics, trends in photonics, photonics

Frontiers in Photonics is a multidisciplinary journal platform targeting high-impact research in the Photonics field at large. As we just commence the third decade of the 21st century, Photonics is becoming more and more a ubiquitous paradigm that sustains the development of a vast spectrum of disciplines. In a landscape so vast, I would like to convey, from my editorial corner, the trepidation and excitement in embracing the scientific mission of Frontiers in Photonics.

In defining what Photonics is, the risk is, undoubtedly, to be trivial and limited. Photonics defines the core science and technology of light in a very broad sense. While this field certainly has an affinity with the general field of optics, with an ancestral origin and very soft (sometimes non-tangible) boundaries, its definition in methods and purpose is relatively recent, and traditionally attributed to Pierre Aigrain (although literature suggests a complex historical genesis) (Balkanski and Lallemand, 1974; Krasnodębski, 2018).

Although the term "photon" became the qualifier of Einstein's quantum of light in the late $20 \mathrm{~s}$ (Lewis, 1926) (and there are accounts that show that the term was already used even before then (Kragh, 2014)), in its accepted definition, the epistemology of "Photonics" is fundamentally broader with a definition being "the science of the harnessing of light. Photonics encompasses the generation of light, the detection of light, the management of light through guidance, manipulation, and amplification, and most importantly, its utilization for the benefit of mankind," and it is directly associated with the invention and diffusion of the laser source (Schawlow and Townes, 1958; Maiman, 1960).

An identifiable trend in the transition between the 20th and the 21st century saw the expansion of Photonics breaking boundaries and cross-pollinating different subject fields. Hence, biophotonics, quantum optics, plasmonics, integrated photonics, optical communications, and terahertz, just to cite a few, represent research areas with sometimes largely distinct sets of established methodologies, theoretical and experimental settings, cohorts of researchers engaged, and, often, distinct research dissemination outlets.

The challenges in those domains, although connected, are indeed distinct. While plasmonics is at the forefront of the effort toward pushing device scaling extremely beyond the wavelength limit (Fernández-Domínguez et al., 2017), integrated photonics is merging with nonlinear optics and complexity in a quest for implementing several complex functionalities (e.g., neuromorphic computing (Burr, 2019), microcombs (Pasquazi et al., 2018), and embedding a large number of degrees of freedom in wavelength-constrained devices. In a further example, quantum optics faces an explosion of new challenges intersecting information technology, communication, imaging, and sensing, with a rising constellation of discoveries, and most importantly, scientific questions, wiping out the classical boundary between physics and engineering (Walmsley, 2015).

Beyond the vital importance of fundamental discoveries, Photonics is, by definition, an applied science. For example, applications in medicine and biology are potent social and economic motivators for developing photonic technologies. Historically speaking, from the invention of the optical microscope (Helden, 2010), medicine and biology underwent countless

Received: 06 November 2020 Accepted: 17 November 20

Peccianti M Grand Challenges in Photonics: Route to Light.

Front. Photonics 1:626728. doi: 10.3389/fphot.2020.626728 transformations related to the exploitation of light. 
Indeed, new solutions for clinical diagnosis and therapies are a central challenge in biophotonics ( $\mathrm{Li}$ and $\mathrm{Pu}, 2019$ ). Many countries recognize Photonics as pivotal in facing major economic and societal challenges, like the green economy, sustainable development, security, inclusive healthcare, inequality, and the digital society, and strive to gain leadership in the field (Light is in the air, 2017). Europe acknowledged Photonics as one of the Key Enabling Technologies (KETs) of the 21 st century and a centerpiece at the core of social development (European Commission, 2020). Notably, the United Kingdom Photonics Leadership Group and the All-Party Parliamentary Group in Photonics and Quantum have in 2020 offered an organic analysis of the industry's dependence on photonics technology and a view about how photonics research will contribute to economic and industrial growth (Photonics Leadership Group, 2020).

The primary mission embraced by Frontiers in Photonics is then to act as a conductor between all those domains, recognizing that the progress of a field is the ultimate research output, above every single exceptional scientific finding. In this sense, highimpact photonic research is not solely based on the report of an extraordinary discovery but also in the way such a discovery

\section{REFERENCES}

M. Balkanski and P. Lallemand (Editors) (1974). "Photonics," in Proceedings of the international meeting of the Délégation générale à la recherche scientifique et technique, Cadarache, France, June 27-29, 1974 (Paris, France: GauthierVillars), 423.

Burr, G. W. (2019). A role for optics in AI hardware. Nature 569, 199-200. doi:10. 1038/d41586-019-01406-0

European Commission. Photonics and our daily life. (2020). Available at: https:// ec.europa.eu/digital-single-market/en/photonics-and-our-daily-life (Accessed August 1, 2020).

Fernández-Domínguez, A. I., García-Vidal, F. J., and Martín-Moreno, L. (2017). Unrelenting plasmons. Nat. Photon. 11, 8-10. doi:10.1038/nphoton.2016.258

Helden, H. V. (2010). The origins of the telescope. 374.

Kragh, H. (2014). Photon: new light on an old name. ArXiv14010293 phys Available at: http://arxiv.org/abs/1401.0293 (Accessed October 25, 2020).

Krasnodębski, M. (2018). Throwing light on photonics: The genealogy of a technological paradigm. Centaurus 60, 3-24. doi:10.1111/1600-0498.12172

Lewis, G. N. (1926). The conservation of photons. Nature 118, 874-875. doi:10. 1038/118874a0

Li, J., and $\mathrm{Pu}, \mathrm{K}$. (2019). Development of organic semiconducting materials for deep-tissue optical imaging, phototherapy and photoactivation. Chem. Soc. Rev. 48, 38-71. doi:10.1039/C8CS00001H

Light is in the air. Nature Photon (2017). 11, 453. doi:10.1038/nphoton.2017.138 ripples in the field, eliciting validation, replication, refinement, confutation, and application.

We designed the broad portfolio of publication types of Frontiers in Photonics to capture this diverse landscape and the established interconnection between domains, offering researchers agile means to reach out, connect to existing scientific work, and showcase successful research endeavors. In such an interdisciplinary field, we aim to define the journal by the quality and impact of its research questions. Frontiers' Research Topics collections, proposed and led by prominent researchers in the field, are a central example, focusing on creating a critical core of experts around a high-impact research topic. In fact, a primary global challenge for science to become "knowledge" is for papers to document transformative discoveries with sufficient detail and accuracy to become tools for other researchers and nonresearchers. This effort is the core target of Frontiers in Photonics.

\section{AUTHOR CONTRIBUTIONS}

The author confirms being the sole contributor of this work and has approved it for publication.

Maiman, T. H. (1960). Stimulated optical radiation in ruby. Nature 187, 493-494 doi:10.1038/187493a0

Pasquazi, A., Peccianti, M., Razzari, L., Moss, D. J., Coen, S., Erkintalo, M., et al. (2018). Micro-combs: a novel generation of optical sources. Phys. Rep. 729, 1-81. doi:10.1016/j.physrep.2017.08.004

Photonics Leadership Group (2020). Future horizons for photonics research 2030 and beyond. Available at: https://photonicsuk.org/wp-content/uploads/2020/ 09/Future-Horizons-for-Photonics-Research_PLG_2020_b.pdf (Accessed September 21, 2020).

Schawlow, A. L., and Townes, C. H. (1958). Infrared and optical masers. Phys. Rev. 112, 1940-1949. doi:10.1103/PhysRev.112.1940

Walmsley, I. A. (2015). Quantum optics: science and technology in a new light. Science 348, 525-530. doi:10.1126/science.aab0097

Conflict of Interest: The author declares that the research was conducted in the absence of any commercial or financial relationships that could be construed as a potential conflict of interest.

Copyright (C) 2021 Peccianti. This is an open-access article distributed under the terms of the Creative Commons Attribution License (CC BY). The use, distribution or reproduction in other forums is permitted, provided the original author(s) and the copyright owner(s) are credited and that the original publication in this journal is cited, in accordance with accepted academic practice. No use, distribution or reproduction is permitted which does not comply with these terms. 